\title{
Distribution OF CXCR4 AND $\gamma$-CATENIN EXPRESSION PATTERN IN BREAST CANCER SUBTYPES AND THEIR RELATIONSHIP TO AXILLARY NODAL INVOLVEMENT
}

\author{
Oya N. Sivrikoz ${ }^{1}$, Latife Doganay ${ }^{2}$, Ulaş Kemal Sivrikoz ${ }^{3}$, Serap Karaarslan ${ }^{1}$, \\ SALAHATTIN M. SANAL ${ }^{4}$
}

\author{
${ }^{1}$ Department of Pathology, Sifa Universitesi Hastanesi, Basmane, Izmir, Turkey \\ 2Department of Pathology, Kent Hastanesi, Cigli, Izmir, Turkey \\ ${ }^{3}$ Family Health Center, Cimentepe, Izmir, Turkey \\ ${ }^{4}$ Division of Oncology, Özel Tinaztepe Hastanesi, Tinaztepe/Buca, Izmir, Turkey
}

\begin{abstract}
Chemokine receptor 4 (CXCR4) and $\gamma$-catenin are known to play an important role in development of metastasis in breast cancer. However, there is not enough information about these biological markers' distribution in different breast cancer subtypes, or their relationship to lymph node metastases in each subtype.

In this study, staining characteristics of CXCR 4 and $\gamma$-catenin were analyzed in each breast cancer subtype and their relationship to lymph node involvement explored. There was a statistically significant relationship between CXCR 4 and certain tumor subtypes $(\mathrm{p}<0.05)$. Basal-like and HER2 enriched tumors showed strong CXCR4 positivity (45.7\%). Furthermore, a significant correlation was discovered between CXCR4 positivity and lymph node involvement $(\mathrm{p}<0.05)$. Among tumor subtypes staining positively with CXCR4, $80 \%$ of basal-like, $90 \%$ of HER2 enriched, and $78.5 \%$ of luminal A showed axillary lymph node involvement. In general, there was a positive relationship between histological grade and CXCR4 expression $(\mathrm{p}=0.004)$. A statistically significant correlation existed between HER2 positivity and $\gamma$-catenin expression $(\mathrm{p}<0.05)$.

Basal-like and HER2 enriched breast cancer subtypes express CXCR 4 staining more often than the other subtypes. Additionally, there is also a positive relationship between lymph node involvement and CXCR4 staining of these subtypes.
\end{abstract}

Key words: breast cancer, CXCR4, $\gamma$-catenin.

\section{Introduction}

Breast cancer is a heterogeneous malignancy that manifests diverse behavior with respect to its prognosis and response to treatment [1]. These differences stem from their genotypic composition [2, 3]. DNA microarray technology helps in identifying these differences, and hence predicting their prognosis and tailoring different treatment approaches [4]. Breast cancer patients have been classified into subtypes on the basis of gene expression analyses [5]. Some of these genotypic features are also identified by immunohistochemical (IHC) staining methods that are widely available and in use on a routine basis. Lately, the disorder has been reclassified on the basis of these molecular features [1]. These features are ER and/or PR status, HER2 expression, CK 5/6 and Ki-67. These features that help us to identify different subtypes are relatively well known to us with respect to their prognostic and therapeutic implications [6]. Nonetheless, we are still in search of other molecular markers within the primary tumor that may help us further in identifying its clinical behavior. 
In this study, we assessed the role of CXCR 4 and $\gamma$-catenin in different breast cancer subtypes and more specifically their relationship with other routinely employed immunohistochemical markers and metastatic involvement of the axillary lymph nodes.

Chemokine receptor 4 (CXCR4) is a G protein coupled transmembrane and cytoplasmic receptor, encoding 352 amino acids, and upon activation is responsible for various inflammatory reactions and adhesion to extracellular matrix molecules $[7,8]$. CXCR 4 is expressed constitutively in a large array of tissues such as heart, lung, liver, and spleen [9-12]. Its ligand is stromal cellderived factor-1a (SDF-1a) [8, 13]. When coupled they play an important role in progression and metastasis of tumors, including breast cancer. The over expression of CXCR 4 in breast cancer is responsible for homing of malignant cells to specific organs and specifically to lymph nodes [14]. Yasuoka et al. discovered that cytoplasmic expression of CXCR 4 has correlated with lymph node involvement [15]. Thus far, it is unclear where the receptor stands with respect to breast cancer subtypes.

$\gamma$-catenin is also known as junction plakoglobin [16]. It is a member of the E-cadherin-catenin family. It is an important component of the submembrane of adherens junctions and desmosomes responsible for cell to cell junction [17]. Low expression of $\gamma$-catenin leads to increased motility of the malignant cells. Conversely, high expression of $\gamma$-catenin leads to decreased motility of the malignant cells and their metastatic potential [18]. Likewise, there is no clear information with respect to where $\gamma$-catenin stands in relation to breast cancer subtypes.

We consecutively investigated the archival tissues of 93 breast cancer patients who had been operated on and performed complete IHC evaluation with respect to the prognostic and therapeutic implications. Furthermore, we searched the IHC staining features of CXCR 4 and $\gamma$-catenin in the primary tumor of different breast cancer subtypes, their complex interrelationship with established markers, and their implications with respect to regional lymph node involvement.

\section{Material and methods}

\section{Study population and IHC staining}

We evaluated a total of 93 operable breast cancer patients diagnosed between 2008 and 2011. Each patient underwent mastectomy/lumpectomy followed by sentinel lymph node biopsy; axillary lymph node dissection was performed upon discovery of nodal metastatic disease. We have kept records of patient age, primary tumor size, and status of lymph node involvement on all the patients. Tumor tissue was evaluated with respect to lymphovascular invasion and histological grade in accordance with modified Bloom-Richardson criteria. Paraffin-embedded blocks from each patient were processed accordingly and stained with hematoxylin eosin for histological evaluation.

All of the IHC staining was performed with a Dako Autostainer 48 Link (Dako, Denmark) for ER (Dako, ready to use) PR (Dako, ready to use), HER2/neu (Dako, dilution 1/400), Ki-67 (Dako, ready to use), as part of the routine evaluation. HER2 was scored on a 0 to 3 + scale using standard criteria. HER 2 was considered negative when the score was 0 or $1+$, positive when $3+$. In patients with HER2 of $2+$, FISH analysis was performed to confirm gene amplification for HER2. Furthermore, as part of our investigation we evaluated the staining patterns of CXCR 4 (Spring Bioscience, dilution 1/50) and $\gamma$-catenin (Novocastra, dilution $1 / 50$ ) in the primary breast cancer tissue of each patient. CK5/6 (Dako, ready to use) staining was performed for triple negative patients.

Patients were classified according to molecular subtypes delineated on the basis of the IHC staining characteristics of the neoplastic tissue. These subtypes were classified according to their staining characteristics for ER, PR, HER2/neu, CK 5/6, and Ki-67. Luminal A is: ER and/or PR (positive), HER2 (negative), $\mathrm{Ki}-67<14 \%$. Luminal B is: ER and/or PR (positive), HER2/neu (negative), Ki-67 $\geq 14 \%$ [19]. Luminal HER2 is: ER and/or PR (positive), HER2 (positive). HER2 enriched is: ER and PR (negative), HER2 (positive). Basal-like is: ER and PR(negative), HER2 (negative), CK5/6 (positive). TNP-nonbasal is: ER and PR (negative), HER2 (negative), CK5/6 (negative). For ease of understanding, basal-like and TNP-nonbasal are both considered within the triple negative category; however, they were analyzed as separate subtypes due to their differences described in this study.

CXCR 4 and $\gamma$-catenin were assessed with respect to their IHC staining properties in both cell membrane and cytoplasm. They were considered positive if there was positive staining in either membrane or cytoplasm [20, 21]. Staining with either CXCR 4 or $\gamma$-catenin was scored according to the percentage of cells that stained positively. Thus, the following designations were made: $0=$ no cells, $1+=1-25 \%$ of cells (weakly positive) (Fig. 1), $2+=26-75 \%$ of cells (Fig. 2) and $3+=$ $>75 \%$ of cells (both strongly positive) (Figs. 3, 4).

\section{Statistical analysis}

The statistical analysis pertaining to data in this study was performed using the SPSS for Windows version 15.0 software package. The relationship between the staining characteristics of each antibody was compared with the clinical and pathologic variables using the Pearson $\chi^{2}$ test. The Kruskal-Wallis test was used to compare between the Ki-67 value and clinicopathological findings. The correlation between lymph node involvement and lymphovascular invasion, CXCR4, 


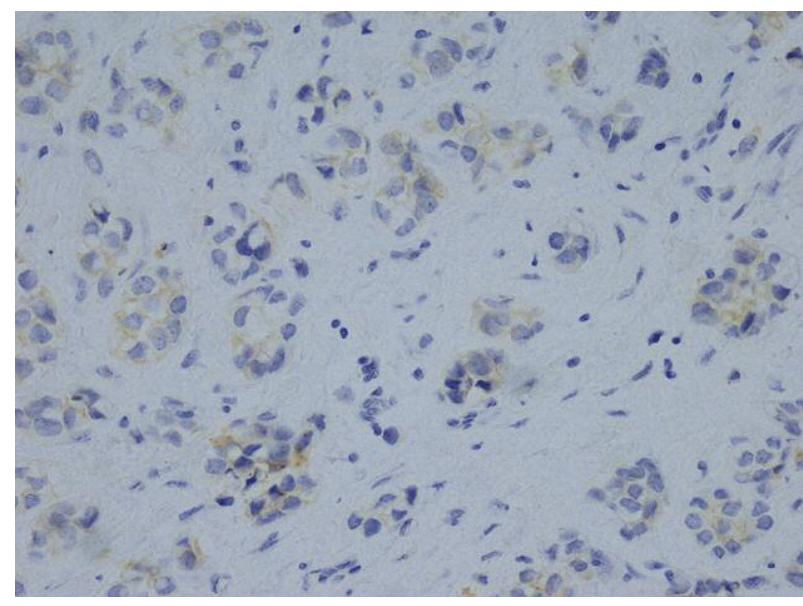

Fig. 1. $\gamma$-catenin staining $1+$ positive $(40 \times)$

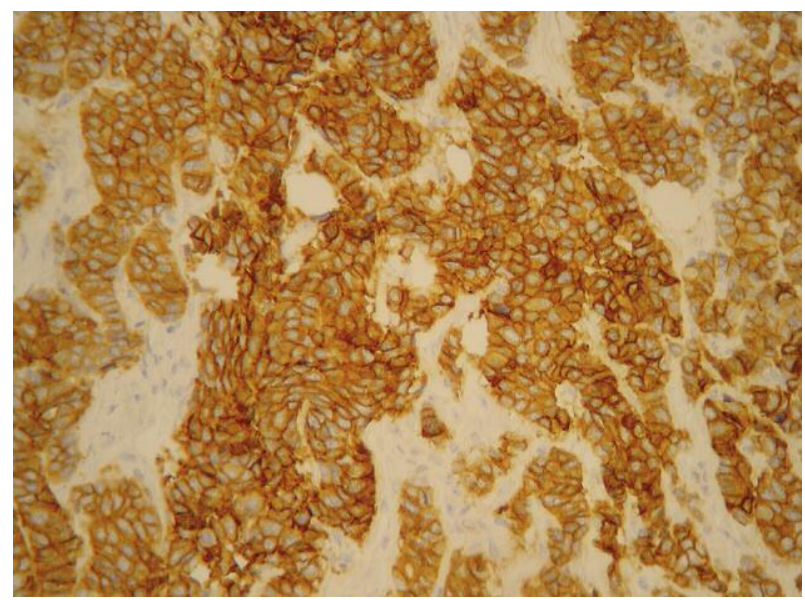

Fig. 3. $\gamma$-catenin staining $3+$ positive $(100 \times)$

$\gamma$-catenin, and HER2 was assessed by logistic regression analysis.

\section{Results}

We analyzed 93 patients between the ages 31 and 99. Their mean age was 54.39 . Of all the patients, $8.6 \%$ were below age $40,48.4 \%$ between ages 40 and 54 , and $43 \%$ above age 54 . The size of the primary tumor was determined according to TNM staging criteria. The largest diameter of the tumor varied between $0.6 \mathrm{~cm}$ and $11 \mathrm{~cm}$. Thus 22 patients had a tumor that measured $<2 \mathrm{~cm}, 61$ patients had a tumor sized $2-5 \mathrm{~cm}$, and 11 patients had a tumor $>5 \mathrm{~cm}$.

Breast cancer subtypes classified according to their molecular features showed the following distribution. Out of 93 patients 39 (41.9\%) were Luminal A, $9(9.7 \%)$ were Luminal B, 15 (16.1\%) were Luminal HER2, 15 (16.1\%) were HER2 enriched, 8 (8.6\%) were basal-like, and 7 (7.5\%) were TNP-nonbasal.

When patients were categorized according to axillary lymph node involvement, 43 patients (46.2\%) did not have any lymph node involvement, 30 patients

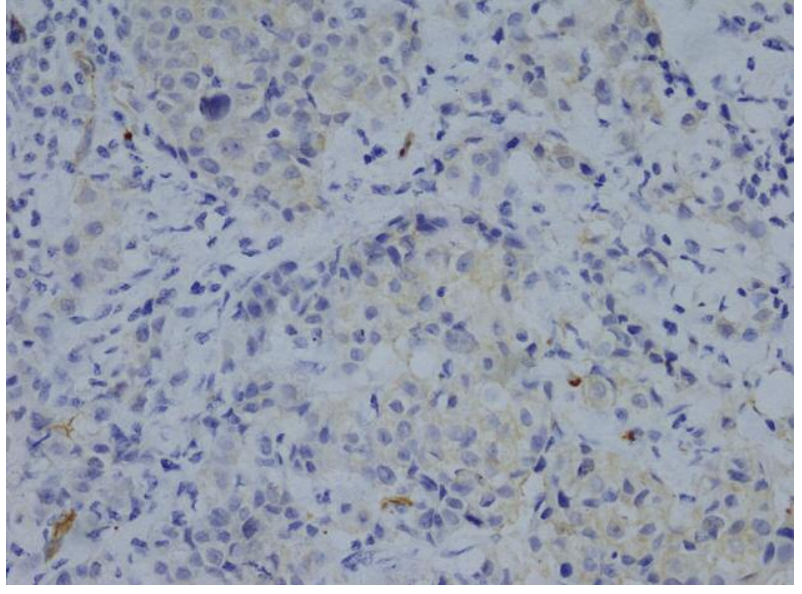

Fig. 2. CXCR4 staining $2+$ positive $(100 \times)$

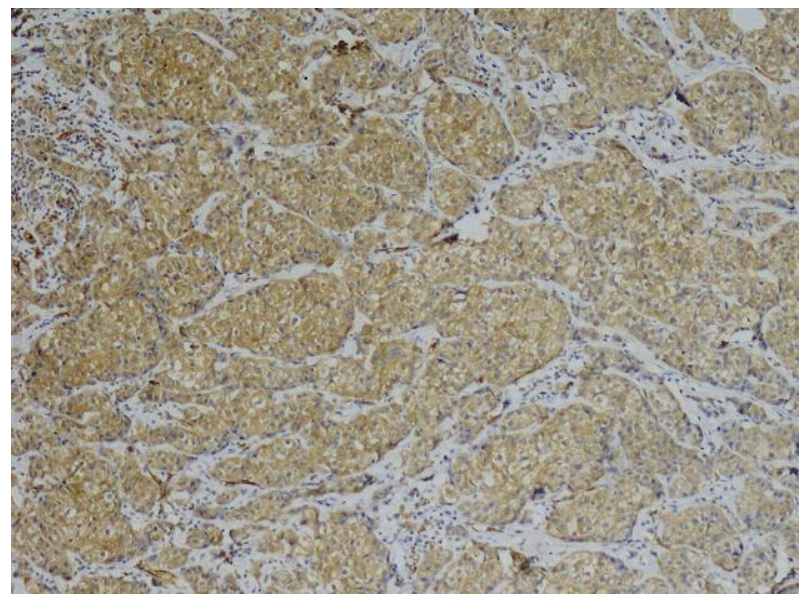

Fig. 4. CXCR4 staining $3+$ positive $(100 \times)$

(32.3\%) had 1-3 lymph nodes, and 20 patients $(21.5 \%)$ had 4 and more lymph nodes involved with metastasis. The distribution of axillary lymph node involvement according to breast cancer subtypes is displayed in Table I. In general, there was not a statistically significant relationship between breast cancer subtypes and lymph node metastasis ( $\mathrm{p}>0.05$ ). Among triple negative patients, $72.5 \%$ of basal-like patients had lymph node involvement, whereas only $14.3 \%$ of TNP-nonbasal patients had lymph node involvement. However, because there were not enough patients in each subtype, the differences were not statistically significant.

A statistically significant correlation existed between CXCR 4 expression and breast cancer subtypes $(\mathrm{p}<0.05)$. In $75 \%$ of basal-like tumors there was positive staining $(2+$ and $3+)$ with CXCR4. Conversely, $71.4 \%$ of TNPnonbasal type tumors showed no staining with CXCR4. In HER2 enriched subtype, $66.7 \%$ showed positive staining $(2+$ and $3+)$. In Luminal A, Luminal B, and Luminal HER2 subtypes, most did not stain at all (0), some showed weak $(1+)$, and very few stained positively $(2+$ and $3+)$ (Table II). 
Table I. Distribution of axillary lymph node involvement according to breast cancer subtypes

\begin{tabular}{|c|c|c|c|c|c|c|c|}
\hline \multirow{2}{*}{$\begin{array}{l}\text { BREAST CANCER } \\
\text { MOLECULAR } \\
\text { SUBTYPES }\end{array}$} & \multicolumn{2}{|c|}{$\begin{array}{l}\text { NO AXILLARY LYMPH } \\
\text { NODE INVOLVEMENT }\end{array}$} & \multicolumn{2}{|c|}{$\begin{array}{c}\text { 1-3 POSITIVE AXILLARY LYMPH } \\
\text { NODE INVOLVEMENT }\end{array}$} & \multicolumn{2}{|c|}{$\begin{array}{l}>4 \text { AXILLARY LYMPH } \\
\text { NODE INVOLVEMENT }\end{array}$} & \multirow[t]{2}{*}{ P-Value } \\
\hline & $\mathrm{N}$ & $\%$ & $\mathbf{N}$ & $\%$ & $\mathrm{~N}$ & $\%$ & \\
\hline Luminal A & 21 & 53.8 & 13 & 33.3 & 5 & 12.8 & \multirow{6}{*}{$>0.05$} \\
\hline Luminal B & 5 & 55.6 & 1 & 11.1 & 3 & 33.3 & \\
\hline Luminal HER2 & 4 & 26.7 & 5 & 33.3 & 6 & 40.0 & \\
\hline HER2 enriched & 4 & 26.7 & 6 & 40.0 & 5 & 33.3 & \\
\hline Basal-like & 3 & 37.5 & 4 & 50.0 & 1 & 12.5 & \\
\hline TNP-nonbasal & 6 & 85.7 & 1 & 14.3 & 0 & 0 & \\
\hline
\end{tabular}

Table II. Relationship of breast cancer subtype with CXCR4 staining pattern

\begin{tabular}{|c|c|c|c|c|c|c|c|c|c|}
\hline \multirow{3}{*}{$\begin{array}{l}\text { BREAST CANCER } \\
\text { MOLECULAR } \\
\text { SUBTYPES } \\
\end{array}$} & \multicolumn{8}{|c|}{ CXCR4 } & \multirow[t]{3}{*}{ P-value } \\
\hline & \multicolumn{2}{|c|}{0} & \multicolumn{2}{|c|}{$1+$} & \multicolumn{2}{|c|}{$2+$} & \multicolumn{2}{|c|}{$3+$} & \\
\hline & $\mathrm{n}$ & $\%$ & $\mathrm{n}$ & $\%$ & $\mathrm{n}$ & $\%$ & $\mathrm{n}$ & $\%$ & \\
\hline Luminal A & 18 & 46.2 & 7 & 17.9 & 6 & 15.4 & 8 & 20.0 & \\
\hline Luminal B & 6 & 66.7 & 2 & 22.2 & 0 & 0 & 1 & 11.1 & \\
\hline Luminal HER2 & 8 & 53.3 & 4 & 26.7 & 0 & 0 & 3 & 20.0 & $<0.05$ \\
\hline HER2 enriched & 2 & 13.3 & 3 & 20.0 & 7 & 46.7 & 3 & 20.0 & \\
\hline Basal-like & 2 & 25.0 & 0 & 0 & 4 & 50.0 & 2 & 25.0 & \\
\hline TNP-nonbasal & 5 & 71.4 & 1 & 14.3 & 0 & 0 & 1 & 14.3 & \\
\hline
\end{tabular}

Table III. Relationship of lymph node metastasis with CXCR4 staining pattern

\begin{tabular}{|c|c|c|c|c|c|c|c|c|c|}
\hline \multirow{3}{*}{$\begin{array}{l}\text { AXILLARY LYMPH } \\
\text { NODE METASTASIS }\end{array}$} & \multicolumn{8}{|c|}{ CXCR4 } & \multirow[t]{3}{*}{$\mathrm{P}$-value } \\
\hline & \multicolumn{2}{|c|}{0} & \multicolumn{2}{|c|}{$1+$} & \multicolumn{2}{|c|}{$2+$} & \multicolumn{2}{|c|}{$3+$} & \\
\hline & $\mathrm{n}$ & $\%$ & $\mathrm{n}$ & $\%$ & $\mathrm{n}$ & $\%$ & $\mathrm{n}$ & $\%$ & \\
\hline 0 & 30 & 69.8 & 6 & 14.0 & 5 & 11.6 & 2 & 4.7 & \\
\hline 1 & 8 & 26.7 & 5 & 16.7 & 6 & 20.0 & 11 & 36.7 & $<0.05$ \\
\hline 2 & 3 & 15.0 & 6 & 30.0 & 6 & 30.0 & 5 & 25.0 & \\
\hline
\end{tabular}

$n$-number of patients; $0-$ no cells stained; $1+-1-25 \%$ of cells stained; $2+-26-75 \%$ of cells stained; $3+->75 \%$ of cells stained

There was a statistically significant relationship between staining characteristics of the primary tumor with CXCR 4 and lymph node involvement (Table III, $\chi^{2}$ $\mathrm{p}<0.05$ ). In $69.8 \%$ of patients without axillary nodal metastatic disease, there was no identifiable CXCR4 staining; strongly positive $(2+$ and $3+)$ staining was identified only in $16.3 \%$. In patients who had axillary metastatic disease, $56 \%$ showed strongly positive $(2+$ and $3+), 22.0 \%$ showed weak $(1+)$, and $22 \%$ showed no staining characteristics.

When the relationship between CXCR4 $(2+$ and $3+)$ staining of the primary tumor subtype and axillary lymph node metastasis was analyzed, basal-like tumors had $80 \%$ (4 out of 6 patients), HER2 enriched had $90 \%$ (9 out of 10 patients), Luminal A had $78.5 \%$ (11 out of 14 patients) axillary lymph node metastasis. Conversely, only one patient in TNP-nonbasal subtype had $(2+$ and $3+)$ positivity and that patient did not show lymph node metastasis (Table II).

Logistic regression analysis was performed to compare the relationship between lymph node involvement and the following characteristics: lymphovascular invasion, histological grade, breast cancer subtype, CXCR4, and positive HER2 expression. Accordingly, in patients with lymph node involvement, $73.2 \%$ showed lymphovascular invasion and $30.6 \%$ positive staining with CXCR4 when analyzed separately. However, $77.7 \%$ demonstrated presence of lympho- 
vascular invasion plus positive CXCR 4 staining when analyzed together. Furthermore, $80.2 \%$ of the patients showed all 3 when positive HER2 expression was added to the preceding two. According to logistic regression analysis, CXCR4 was an independent factor showing a correlation with lymph node involvement.

In general, there was a statistically significant relationship between histological grade and CXCR4 staining $(\mathrm{p}<0.05)$, relevant for all the subtypes. Among grade 1 tumors $28.9 \%$, grade 2 tumors $37.8 \%$, and grade 3 tumors $72.7 \%$ showed strong positivity $(2+$ and $3+)$ with CXCR 4 staining. Thus, there was a corresponding CXCR4 positivity with increasing tumor grade.

There was a direct relationship between histological grade and lymph node involvement as well. Specifically, there was a distinct difference comparing histological grade 1 with grade 2 and 3 tumors; however, the rate of lymph node involvement was lower in grade 3 tumors than in grade 2 tumors.

There was no relationship between the patient age, size of primary tumor, $\gamma$-catenin, Ki-67 staining characteristics, and lymph node involvement.

There was a corresponding linear relationship with increasing $\gamma$-catenin staining score and HER 2 positivity. In reference to breast cancer subtypes, $73.3 \%$ of HER2 enriched and $100 \%$ of Luminal HER 2 patients showed strongly positive staining $(2+$ and $3+)$ with $\gamma$-catenin. Conversely, there was either no or at the most weak $(1+)$ staining in $60 \%$ of patients in Luminal A and B subtypes.

\section{Discussion}

Breast carcinoma is a heterogeneous malignant disorder [1]. Originally, different subtypes of breast cancers were identified on the basis of gene profiling technology $[2,3]$. Later, investigators demonstrated that 6 different breast cancer subtypes can also be identified on the basis of their IHC staining characteristics, with each subtype having different prognostic and therapeutic implications $[1,19]$.

Gene expression profiling studies demonstrated that each biologically (molecularly) different breast cancer subtype has a distinct prognosis [1]. Basal-like and HER2 enriched subtypes have inferior disease-free and overall survival than the luminal subtypes. Additionally, patients with Luminal B subtype have worse prognosis than the Luminal A subtype [19, 22]. In $30 \%$ of Luminal B subtype patients, over-expression of HER2 is demonstrated [19]. These patients are reclassified as Luminal-HER2 since they are candidates for treatment with trastuzumab, a humanized monoclonal antibody against HER2 receptor [23].

In this study, we categorized our patients in accordance with their staining characteristics for ER, PR, HER2, CK5/6, and Ki-67 and classified them into 6 subtypes as defined by [1]. In each subtype, we an- alyzed the relationship between lymph node involvement and CXCR 4 as well as $\gamma$-catenin staining patterns. Thus far, we have not seen any study that compared lymph node involvement in each subtype with CXCR4 and $\gamma$-catenin staining characteristics. Recently, investigators described an important link between breast cancer subtypes and lymph node involvement [1]. Conversely, in our study we were not able to demonstrate a significant relationship between breast cancer subtype and axillary lymph node metastasis. Nonetheless, there was a clear difference among triple negative patients with $72.5 \%$ metastatic lymph node involvement in basal-like and $14.3 \%$ in TNP-nonbasal subgroups. This was not statistically significant in this study due to the limited number of patients. Because of such a difference in lymph node involvement, albeit not statistically significant, these TNP tumor subgroups have not only distinct molecular characteristics, they also have different prognoses [24, 25]. Thus, we believe it is well justified that even though they are classified as triple negative breast cancer, they should continue to be categorically separated as basal-like and TNP-nonbasal. Hence, further investigation is warranted to better identify such differences.

There are numerous studies that have shown a direct relationship between CXCR4 staining characteristics and lymph node involvement [7, 14, 15, 26]. In our study we also discovered a direct relationship between lymph node involvement and histological grade, HER2, and CXCR4 staining characteristics, whereas there was no such relationship with $\gamma$-catenin, primary tumor size, patient age, and subtypes of breast cancer. Kato et al. investigated the relationship between CXCR4 staining characteristics and lymph node involvement in breast cancer patients and discovered that focal staining pattern was more often associated with lymph node involvement than diffuse staining pattern [14]. In our study, we also discovered a distinct relationship between the presence of lymph node involvement and CXCR4 staining. This was very similar to the observations of Kato et al., showing a relationship between staining pattern and number of lymph nodes involved. Although lymphovascular invasion in the primary tumor is the most significant predictor of lymph node involvement, CXCR 4 by itself predicts lymph node involvement in $30 \%$ of patients.

We also found a significant relationship between breast cancer subtypes and staining characteristics for both $\gamma$-catenin and CXCR4. Thus far, we have found a single article addressing the relationship between CXCR4 and molecular subtypes of breast cancer [27]. Zhand et al. investigated the relationship between CXCR4 and 5 different molecular subtypes of breast cancer. They discovered a marked difference between Luminal A and basal-like tumors with respect to their staining pattern [27]. In basal-like tumors CXCR 4 protein expression was higher than Luminal A tumors. In our 
study, we found that $75 \%$ of basal-like tumors showed intense staining $(2+$ and $3+)$, whereas only $14.3 \%$ of TNP-nonbasal tumors (1 patient) showed such a staining pattern, and the rest did not show any staining at all. We believe these observations, along with their different propensity for lymph node involvement, are further evidence supporting the notion that basal-like and TNP-nonbasal tumors are distinct groups of tumors and should be assessed separately with respect to their prognostic and therapeutic implications [24, 25]. In contrast, Luminal A, Luminal B, and Luminal HER2 tumors showed either no or weak staining at the most.

We did not observe a statistically significant relationship between breast cancer subtypes and histological grade, done in accordance with the modified Bloom-Richardson grading system. Nevertheless, there was a difference when Luminal A patients were compared to triple negative patients (basal-like and TNPnonbasal together), triple negative patients having a higher tumor grade. Even though there was not a significant difference between the subtypes with respect to lymph node involvement and extent of lymphovascular invasion, triple negative breast cancer patients are thought to have worse prognosis on the basis of their higher histological grade and Ki67 value [24, 25]. Thus, when triple negative breast cancer patients are compared with the other subtypes, there are reports indicating that these tumors take a clinically aggressive course.

In contrast to published results, we found no relationship between nodal involvement and breast cancer subtypes, although there was an independent relationship between lymph node involvement and CXCR4 staining.

Because there is a demonstrated relationship between HER2 oncogene product and $\gamma$-catenin complex in general $[28,29]$, we discovered a similar relationship in patients with HER2 enriched and Luminal HER2, showing co-existence of both these biological markers in tumor specimens of these patients $(\mathrm{p}=0.000)$.

Recently, FISH analysis has been in use to identify HER2 gene amplification [19]. Because of the close relationship identified between HER2 and $\gamma$-catenin, the latter may be helpful in determining presence of HER2 gene amplification without the need for an expensive FISH test. We believe this is an interesting observation warranting further investigation.

Axillary lymph node dissection and even sentinel lymph node biopsy carry significant morbidity [30]. Efforts are undertaken to predict presence of axillary lymph node involvement in order to minimize the need for surgical intervention. It is premature to recommend that CXCR 4 be used to identify patients without any lymph node involvement. Nonetheless, CXCR4 in combination with HER 2 and lymphovascular invasion may encourage similar efforts to discover novel biological markers in making such a decision, especially in patients showing no clinical evidence of lymph node involvement with physical examination and ultrasonographic evaluation of the axillary region.

The authors declare no conflict of interest.

\section{References}

1. Voduc KD, Cheang MCU, Tyldesly, S, et al. Breast cancer subtypes and the risk of local and regional relapse. J Clin Oncol 2010; 28: 1684-1691.

2. Perou CM, Sørlie T, Eisen MB, et al. Molecular portraits of human breast tumors. Nature 2000; 406: 747-752.

3. van 't Veer LJ, Dai H, van de Vijver MJ, et al. Gene expression profiling predicts clinical outcome of breast cancer. Nature 2002; 415: 530-536.

4. Brenton JD, Carey LA, Ahmed AA, Caldas C. Molecular classification and molecular forecasting of breast cancer: Ready for clinical application? J Clin Oncol 2005; 23: 7350-7360.

5. Sorlie T, Perou CM, Tibshirani R, et al. Gene expression patterns of breast carcinomas distinguish tumor subclasses with clinical implications. Proc Natl Acad Sci U S A 2001; 98: 1086910874.

6. Adamczyk A, Niemiec J, Ambicka A, et al. Expression of ER/PR/HER2, basal markers and adhesion molecules in primary breast cancer and in lymph nodes metastases: a comparative immunohistochemical analysis. Pol J Pathol 2012; 63: 228-234.

7. Hiller D, Chu QD. CXCR4 and axillary lymph nodes: review of a potential biomarker for breast cancer metastasis. Int J Breast Cancer 2011; 2011: 420981.

8. Liang Z, Yoon Y, Votaw J, et al. Silencing of CXCR4 blocks breast cancer metastasis. Cancer Res 2005; 65: 967-971.

9. Li N, Guo W, Shi J, et al. Expression of the chemokine receptor CXCR4 in human hepatocellular carcinoma and its role in portal vein tumor thrombus. J Exp Clin Cancer Res 2010; 29: 156.

10. Umemoto E, Otani K, Ikeno T, et al. Constitutive plasmacytoid dendritic cell migration to the splenic white pulp is cooperatively regulated by CCR7- and CXCR4-mediated signaling. J Immunol 2012; 189: 191-199.

11. Segret A, Rücker-Martin C, Pavoine C, et al. Structural localization and expression of CXCL12 and CXCR 4 in rat heart and isolated cardiac myocytes. J Histochem Cytochem 2007; 55: 141150.

12. Ueland J, Yuan A, Marlier A, et al. A novel role for the chemokine receptor Cxcr4 in kidney morphogenesis: an in vitro study. Dev Dyn 2009; 238: 1083-1091.

13. Hernandez L, Magalhaes MA, Coniglio SJ, et al. Opposing roles of CXCR 4 and CXCR7 in breast cancer metastasis. Breast Cancer Res 2011; 13: R128.

14. Kato M, Kitayama J, Kazama S, Nagawa H. Expression pattern of CXC chemokine receptor- 4 is correlated with lymphnode metastasis in human invasive ductal carcinoma. Breast Cancer Res 2003; 5: R144-R150.

15. Yasuoka H, Tsujimoto M, Yoshidome K, et al. Cytoplasmic CXCR4 expression in breast cancer: induction by nitricoxide and correlation with lymphnode metastasis and poor prognosis. BMC Cancer 2008; 8: 340.

16. Lai YH, Cheng J, Cheng D, et al. SOX 4 interacts with plakoglobin in a Wnt3a-dependent manner in prostate cancer cells. BMC Cell Biol 2011; 12: 50.

17. Holen I, Whitworth J, Nutter F, et al. Loss of plakoglobin promotes decreased cell-cell contact, increased invasion, and breast cancer cell dissemination in vivo. Breast Cancer Res 2012; 14: R86. 
18. Bailey CK, Mittal MK, Misra S, Chaudhuri G. High motility of triple-negative breast cancer cells is due to repression of plakoglobin gene by metastasis modulator protein SLUG. J Biol Chem 2012; 287: 19472-19486.

19. Cheang MC, Chia SK, Voduc D, et al. Ki67 index, HER2 status, and prognosis of patients with luminal B breast cancer. J Natl Cancer Inst 2009; 101: 736-750.

20. Su YC, Wu MT, Huang CJ, et al. Expression of CXCR4 is associated with axillary lymph node status in patients with early breast cancer. Breast 2006; 15: 533-539.

21. Bukholm IK, Nesland JM, Børresen-Dale AL. Re-expression of E-cadherin, alpha-catenin and beta-catenin, but not of gammacatenin, in metastatic tissue from breast cancer patients. J Pathol 2000; 190: 15-19.

22. Lavasani MA, Moinfar F. Molecular classification of breast carcinomas with particular emphasis on "basal-like" carcinoma: a critical review. J Biophotonics 2012; 5: 345-366.

23. Kobayashi T, Iwaya K, Moriya T, et al. A simple immunohistochemical panel comprising 2 conventional markers, Ki67 and $\mathrm{p} 53$, is a powerful tool for predicting patient outcome in luminal-type breast cancer. BMC ClinPathol 2013; 13: 5.

24. Dent R, Trudeau M, Pritchard KI, et al. Triple-negative breast cancer: clinical features and patterns of recurrence. Clin Cancer Res 2007; 13 (15 Pt 1): 4429-4434.

25. Prat A, Adamo B, Cheang MC, et al. Molecular characterization of basal-like and non-basal-like triple-negative breast cancer. Oncologist 2013; 18: 123-133.

26. Razmkhah M, Jaberipour M, Safaei A, et al. Chemokine and chemokine receptors: a comparative study between metastatic and nonmetastatic lymph nodes in breast cancer patients. Eur Cytokine Netw 2012; 23: 72-77.

27. Zhang M, Liu HX, Teng XD, et al. The differences in CXCR4proteinexpression are significant for the five molecular subtypes of breast cancer. Ultrastruct Pathol 2012; 36: 381-386.

28. Kanai Y, Ochiai A, Shibata T, et al. c-erbB-2 gene product directly associates with beta-catenin and plakoglobin. Biochem Biophys Res Commun 1995; 208: 1067-1072.

29. Li Y, Yu WH, Ren J, Chen W, et al. Heregulin targets gamma-catenin to the nucleolus by a mechanism dependent on the DF3/MUC1 oncoprotein. Mol Cancer Res 2003; 1: 765-775.

30. Reyal F, Belichard C, Rouzier R, et al. Non-sentinel lymph node metastasis prediction in breast cancer with metastatic sentinel lymph node: impact of molecular subtypes classification. PLoS One 2012; 7: e47390.

\title{
Address for correspondence
}

\author{
Salahattin M. Sanal MD \\ Division of Medical Oncology \\ Özel Tinaztepe Hastanesi \\ Ahmet Piristina Bulvari 203/27 Sk. No: 12 \\ Tinaztepe/Buca, Izmir, Turkey 35160 \\ tel. $+90(232) 4541414$ \\ fax +90(232) 4541516 \\ e-mail: sanal_salahattin@yahoo.com
}

